Die Herren Dr. Kre y und Dr. Scheithauer berichten über den Jubiläumsfonds. Es wird mitgeteilt, daß durch Beschluß des Vorstandsrats an Stelle des verstorbenen Herrn Dr. Pr üs s ing Herr Dr. Kr e y zum Kurator des Jubiläumsfonds ernannt worden ist.

3. Der Haushaltungsplan des Vereins für 1916 wird von Dr. S cheitha u e r erläutert und von der Versammlung genehmigt.

4. Vorstandswahlen: An Stelle des satzungsgemäß ausscheidenden Vorsitzenden, Direktor Dr. Her $\mathrm{m}$ a $\mathrm{n} \mathrm{K} \mathrm{r}$ e y, Halle a. S., der eine Wiederwahl ablehnt, wird Herr Dr. T h e od o r D i e h l, Berlin, einstimmig gewählt. Der satzungsgemäß ausscheidende $\mathrm{Schatz}$ -

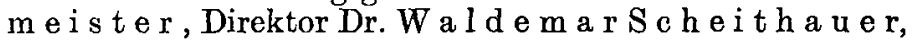
Halle a. S., wird einstimmig wiedergewählt. Es haben ferner für den Schlu $\beta$ des laufenden Jahres ihre V o r s t a n d s ä $\mathrm{m}$ t e $\mathrm{r}$ niedergelegt: der stellvertretende Vorsitzende, Geh. Reg.-Rat Prof. Dr. M a x D e l b r ü c k, Berlin, und der Beisitzer Dr. A l bert J a e g e r, Koblenz, letzterer wegen Veränderung seiner Berufsstellung, die die Voraussetzung seiner Wahl als Vertreter der angestellten Chemiker nicht mehr gegeben erscheinen läßt. An ihrer Stelle werden die Herren Prof. Dr. Ka r l Lint ner, München, und Dr. Fritz Jander, Goldschmieden bei Deutsch-Lissa, einstimmig in den Vorstand gewählt.

Dr. Die h l nimmt die Wahl zum Vorsitzenden mit Dank an. Dr. Scheith a u e nimmt die Wiederwahl mit Dank an.

$\mathrm{Zu}$ Rechnungsprïfern werden die Herren $\mathrm{P}$ a u $1 \mathrm{~K}$ o b e und Direktor Dr. J o h a n e s $\mathrm{N}$ e u g e b a u e r einstimmig wiedergewählt.

5. Als Ort der Hauptversammlung 1916 wird Braunschweig bestimmt. Festsetzung des Zeitpunktès der Versammlung wird dem Vorstand überlassen.

6a. Der Bericht über die Entwicklung der Vereinszeitschrift im Jahre 1914 sowie über den bisherigen Verlauf des Jahres 1915 liegt gedruckt vor; er wird zur Kenntnis genommen.

6b. Prof. R a s s ow berichtet über die Tätigkeit der Anzeigenverwaltung.

7. Der gedruckt vorliegende Bericht über die Statistik der Chemiker und Chemiestudierenden wirả zur Kenntnis genommen.

3. Der Bericht über die Stellenvermittlung wird ge nehmigt, er liegt gedruckt vor.

An einer Aussprache über die Gehälter und die Stellung der Chemiker beteiligen sich die Herren Ple $\mathrm{nsk}$ e, Ma-

\section{Die Unterteilung von Steingutglasuren in Fritte und Versatz und ihre Beziehung zu Glasurfehlern.}

Von Dr. H. Hankont, Velten i. M.

(Eingeg. 14./9. 1915.)

Als wichtigstes theoretisches Hilfsmittel für die Beurteilung von Glasuren dient die sogenannte Segerformel, also die Darstellung des molekularen Aufbaues unter Gegenüberstellung von Basen, Säuren und der Tonerde. Diese wird immer aus den sämtlichen zur Verwendung gelangenden Materialien errechnet, also aus dem theoretischen Endzustand der Glasur. Steingutglasuren werden aber stets durch Unterteilung des Gesamtversatzes in Fritte und Versatz gewonnen, so daß beim Prozeß des Glattbrandes die mit der Fritte erschmolzene Glasur, die von der Endglasur mehr orler weniger abweicht, mit den im Ausgangsstadium befindlichen Versatzmaterialien in Reaktion tritt, um die Endglasur zu bilden. Man hat nun vielfach angenommen, $\mathrm{da} ß$, da es sich um das gleiche Endprodukt handelt, die Art dieser Unterteilung ohne Belang sei, während doch viel Grund zu der Annahme vorliegt, daß es zum mindesten für den Verlauf der Reaktion bzw. Schmelzprozeß von Wichtigkeit ist, welche Reagenzien aufeinander einwirken, d. h. wie die Unterteilung geschehe. Ältere Angaben über gebräuchliche Steingutglasuren geben entsprechend ihrer empririschen Natur ein Bild systemloser Vielseitigkeit in bezug auf diese Unterteilung ${ }^{1}$ ). Mit der Forderung, wegen

\footnotetext{
1) S. z. B. Kerl, Handbuch d. ges. Tonwarenindustrie S. 1154 u. f.
}

kowka, Hempel, Freseni us, $\mathrm{H}$ esse und cier Vorsitzende.

9. Dr. Scheith a u e r berichtet über die Entwicklung der Hilfskasse und ihre Tätigkeit im vergangenen Jahre. Dr. J o r d a n wird als Mitglied des Kuratoriums der Hilfskasse wiedergewählt und nimmt die Wahl an.

9b. Dr. S c h e it h a u er erstattet den Bericht über die Tätigkeit der Kriegshilfe im vergangenen Jahre und über den bisherigen Verlauf des Jahres 1915.

10. Der Bericht des Herrn Prof. Osterrieth über öie Tätigkeit der Rechtsauskunftsstelle, der gedruckt vorliegt, wird zur Kenntnis genommen. Dazu nimmt das Wort Herr Dr. M a k ow ka und der Berichterstatter.

11a. Der gedruckt vorliegende Bericht des Herrn Prof. Osterrieth über die Tätigkeit des Sozialen Ausschusses wird genehmigt.

$11 \mathrm{~b}$. An Stelle der ausscheidenden angestellten Chemiker, der Herren Dr. J a $\mathrm{nd} \mathrm{e} \mathrm{r}$ und Dr. Ul r i ch, werden die Herren Dr. Bäre nf änger, Kiel, und Dr. H e i m, München, gewählt. An Stelle des ausscheidenden unabhängigen Chemikers Herrn Dr. D i ehl wird Herx Dr. $\mathbf{Q}$ u in c k e gewählt, und Herr Dr. M e y e r wird wiedergewählt. Die beiden letzteren Herren sind anwesend und nehmen die Wahl an.

12. Der Bericht über den Ausschuß zur Wahrung der gemeinsamen Interessen des Chemikerstandes liegt gedruckt vor; er wird zur Kenntnis genommen.

13. Der gedruckt vorliegende Bericht über die Tätigkeit des deutschen Ausschusses für naturwissenschaftlichen und mathematischen Unterricht wird zur Kenntnis genommen.

14. Der Bericht über den Verein zur Förderung chemischer Forschung wird genehmigt.

15. Der Vorsitzende macht Mitteilung über die von der Geschäftsstelle geplante Errichtung einer Hinterlegungsstelle für Geheimverfahren.

Schluß der Sitzung 11,30 Uhr.

Der Protokollführer: B. R a s sow.

Dr. H a n s Alexander. Dr. Busch. Dr. M. K. $\mathrm{H}$ off $\mathrm{mann}$. Prof. Joh annes Mülier. Dr. E. Stern. Prof. Dr. Precht. Prof. E. Erdmann.

Nach der Sitzung vereinigte ein gemeinsames Mittag. essen bei Adlon noch einmal die Teilnehmer auf einige Stunden in zwanglosem Beisammensein, in dessen Verlauf Herr Geheimrat Prof. Dr. R. Möhlau dem Dank der Versammlung für die aufopfernde Tätigkeit des mit Ende des Jahres aus seinem Amte ausscheidenden Vorsitzenden mit einem dreimaligen Hoch Ausdruck verlieh.

seiner Gesundheitsschädlichkeit das Bleioxyd aus dem Versatz zu entfernen und durch Einbezug in die Fritte ungefährlich zu machen, wurde zuerst die Beschäftigung mit der Frage allgemein, und die praktischen Wirkungen solcher Änderungen in der Unterteilung waren derart, daß noch heute viele Betriebe diese Änderung nicht vollzogen haben. Trotzdem hat man mit der fortschreitenden theoretischen Behandlung der Glasuren geglaubt, diese Frage vernachlässigen zu können, und fast allgemein wird bei Wiedergabe von Glasurformeln über die Unterteilung nichts gesagt, und

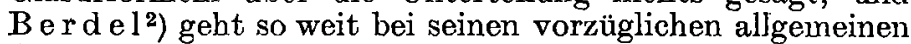
Angaben über Steingutglasuren, diese dahingehend zu schematisieren, daß der Gesamtglasur zur Gewinnung der Fritte immer nur 0,05-0,10 Äquivalente Kaolin als Versatz entzogen werden. So gibt auch $\mathrm{D}$ or $\mathrm{f} n \mathrm{r}^{3}$ ) in seinen so wichtigen Mitteilungen über bleifreie Glasuren leider nicht an, wie er seine Glasur in Fritte und Versatz teilte, und erledigt diese Frage mit den Worten: ,,Es soll nur Segerformel und Hauptversatz Erwähnung finden, da es den keramisch vorgebildeten Fachmann keinerlei Schwierigkeiten bereiten kann, die hierzu passende Fritte und Mühlversätze auszurechnen."

Die in dieser Arbeit niedergelegten Erfahrungen zeigen aber, daß die Unterteilung nicht nur nicht gleichgültig ist, sondern daß Glasuren von gleicher Endformel, aber durch verschiedene Art der Unterteilung gewonnen, derart stark,

2) B e rde ], Anleitung zu keramischen Versuchen S. 40.

3) Sprechsaal 47, 390 [1914]. 
sowohl in ihrem Verhalten während des Glattbrandes, wie sogar in ihrem endgültigen Aussehen abweichen können, daß die eine ganz unbrauchbar, die andere vorzüglich ist. Ehe Einzelangaben gemacht werden, sei vorausgeschickt, da $B$ alle Ergebnisse im Großbetrieb, also an größeren Mengen und wiederholt gewonnen wurden, und es sich dabei um den Glattbrand im Rundofen mit überschlagender Flamme und Rostfeuerung mit Handbeschickung handelte. Zum Vergleich wurden Brände in der Muffel und im Ringofen berangezogen. Die Glasurbereitung wurde in der im Großbetrieb üblichen Weise vorgenommen, und im allgemeinen wurden die Glasuren auf Wandplatten, die mit der Glasurseite sich gegenübcrstehend in Kapseln eingefüllt wurden, aufgeschmolzen. Zum Vergleich wurden auch Gefäße mit ihnen glasiert, es zeigte sich aber, daß die große ebene Fläche der Wandplatten am besten zur Beobachtung des Aussehens und aller Erscheinungen der Glasuroberfläche geeignet ist.

Zunächst sind in der folgenden Tabelle alle zur Untersuchung herangezogenen Glasuren zusammengestellt unter Angabe ihrer Segerformel, ihrer prozentualen Zusammensetzung aus Fritte und Versatz, ihrer prozentualen Gesamtzusammensetzung und endlich unter Angabe der Segerformel ihrer Fritte oder Fritten.

Der leitende Gedanke bei Aufbau der Glasuren war zunächst der, für einen niederen Glattbrand von etwa Kegel

Tabelle der untersuchten Glasuren.

\begin{tabular}{|c|c|c|c|c|c|c|c|}
\hline $\begin{array}{l}\text { Bezeich- } \\
\text { nung der } \\
\text { Glasur }\end{array}$ & $\begin{array}{l}\text { Art der Unterteilung } \\
\text { in } \%\end{array}$ & $\begin{array}{l}\text { Seger } \\
\text { der Ges }\end{array}$ & $\begin{array}{l}\text { ormel } \\
\text { mtglasur }\end{array}$ & Segerforme & der Fritten & $\begin{array}{r}\text { Gesamtzusa } \\
\text { in }\end{array}$ & $\%$ \\
\hline$V$ & $\begin{array}{c}32,2 \% \text { Fritte } \mathrm{E} \\
37,7 \% \text {. } 155 \\
6,5 \% \text { Feidspat } \\
11,3 \% \text { Kreide } \\
12,3 \% \text { Kaolin }\end{array}$ & $\begin{array}{l}0,54 \mathrm{CaO} \\
0,02 \mathrm{Na}_{2} \mathrm{O} \\
0,16 \mathrm{~K}_{2} \mathrm{O} \\
0,27 \mathrm{PbO} \\
0,99\end{array}$ & $\begin{array}{l}2,65 \mathrm{SiO}_{2} \\
0,37 \mathrm{~B}_{2} \mathrm{O}_{3} \\
0,35 \mathrm{Al}_{2} \mathrm{O}_{3}\end{array}$ & $\begin{array}{ll} & \text { Fritte } 155 \\
0,07 \mathrm{CaO} & 4,10 \mathrm{SiO}_{2} \\
0,03 \mathrm{Na}_{2} \mathrm{O} & 0,90 \mathrm{~B}_{2} \mathrm{O}_{3} \\
0,26 \mathrm{~K}_{2} \mathrm{O} & 0,26 \mathrm{Al}_{2} \mathrm{O}_{3} \\
0,64 \mathrm{PbO} & \\
1,00 & \end{array}$ & \begin{tabular}{ll}
\multicolumn{2}{c}{ Fritte $\mathrm{E}$} \\
$0,42 \mathrm{CaO}$ & $3,48 \mathrm{SiO}_{2}$ \\
$0,18 \mathrm{~K}_{2} \mathrm{O}$ & $0,4 \pm \mathrm{B}_{2} \mathrm{O}_{3}$ \\
$0,03 \mathrm{Na}_{2} \mathrm{O}$ & $0,23 \mathrm{Al}_{2} \mathrm{O}_{3}$ \\
$\frac{0,37}{\mathrm{PbO}}$ & \\
1,00 & \\
\end{tabular} & $\begin{array}{c}9,3 \mathrm{CaO} \\
18,7 \mathrm{PbO} \\
4,6 \mathrm{~K}_{2} \mathrm{O} \\
0,3 \mathrm{Na}_{2} \mathrm{O}\end{array}$ & $\begin{array}{c}49,0 \mathrm{SiO}_{2} \\
7,6 \mathrm{~B}_{2} \mathrm{O}_{3} \\
10,6 \mathrm{Al}_{2} \mathrm{O}_{3}\end{array}$ \\
\hline$G_{1}$ & $\begin{array}{c}63,5 \% \text { Fritte } \mathrm{W}_{9} \\
16,4 \% \text { \% } \mathrm{W}_{7} \\
11,4 \% \text { Kaolin } \\
8,8 \% \text { Quarz }\end{array}$ & $\begin{array}{l}0,52 \mathrm{CaO} \\
0,10 \mathrm{Na}_{2} \mathrm{O} \\
0,08 \mathrm{~K}_{2} \mathrm{O} \\
\frac{0,30}{1,00} \mathrm{PbO}\end{array}$ & $\begin{array}{l}2,13 \mathrm{SiO}_{2} \\
0,36 \mathrm{~B}_{2} \mathrm{O}_{3} \\
0,21 \mathrm{Al}_{2} \mathrm{O}_{3}\end{array}$ & 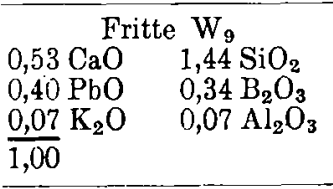 & \begin{tabular}{ll}
\multicolumn{2}{r}{ Fritte $\mathrm{W}_{7}$} \\
$0,48 \mathrm{CaO}$ & $1,46 \mathrm{SiO}_{2}$ \\
$0,40 \mathrm{Na}_{2} \mathrm{O}$ & $0,40 \mathrm{~B}_{2} \mathrm{O}_{3}$ \\
$\frac{0,12 \mathrm{~K}_{\mathbf{2}} \mathrm{O}}{1,00}$ & $0,12 \mathrm{Al}_{2} \mathrm{O}_{3}$
\end{tabular} & $\begin{array}{c}10,2 \mathrm{CaO} \\
23,4 \mathrm{PbO} \\
2,2 \mathrm{~K}_{2} \mathrm{O} \\
2,7 \mathrm{Na}_{2} \mathrm{O}\end{array}$ & $\begin{array}{c}45,0 \mathrm{SiO}_{2} \\
8,9 \mathrm{~B}_{2} \mathrm{O}_{3} \\
7,5 \mathrm{Al}_{2} \mathrm{O}_{3}\end{array}$ \\
\hline$W_{\underline{a}}$ & $\begin{array}{cl}72,0 \% & \text { Fritte } 155 \\
7,6 \% & \text { Feldspat } \\
15,4 \% & \text { Kreide } \\
3,6 \% & \text { Kaolin } \\
1,8 \% \text { Mennige }\end{array}$ & $\begin{array}{l}0,52 \mathrm{CaO} \\
0,01 \mathrm{Na}_{2} \mathrm{O} \\
0,16 \mathrm{~K}_{2} \mathrm{O} \\
0.31 \mathrm{PbO} \\
\frac{1,00}{}\end{array}$ & $\begin{array}{l}2,14 \mathrm{SiO}_{2} \\
0,39 \mathrm{~B}_{2} \mathrm{O}_{3} \\
0,20 \mathrm{Al}_{2} \mathrm{O}_{3}\end{array}$ & $\begin{array}{l}\text { Fritte } 155 \\
\text { siehe bei } V\end{array}$ & & $\begin{array}{c}10,0 \mathrm{CaO} \\
23,5 \mathrm{PbO} \\
5,2 \mathrm{~K}_{2} \mathrm{O} \\
0,2 \mathrm{Na}_{2} \mathrm{O}\end{array}$ & $\begin{array}{c}44,8 \mathrm{SiO}_{2} \\
9,3 \mathrm{~B}_{2} \mathrm{O}_{3} \\
7,0 \mathrm{Al}_{2} \mathrm{O}_{3}\end{array}$ \\
\hline $\mathrm{G}_{4}$ & $\begin{aligned} 73,0^{\circ} \% & \text { Fritte } \mathrm{G}_{4} \\
7,6 \% & \text { Feldspat } \\
15,7 \% & \text { Kreide } \\
3,7 \% & \text { Kaolin }\end{aligned}$ & $\begin{array}{l}0,52 \mathrm{CaO} \\
0,01 \mathrm{Na}_{2} \mathrm{O} \\
0,16 \mathrm{~K}_{2} \mathrm{O} \\
\frac{0,31}{1,00} \mathrm{PbO}\end{array}$ & $\begin{array}{l}2,16 \mathrm{SiO}_{2} \\
0,39 \mathrm{~B}_{2} \mathrm{O}_{3} \\
0,21 \mathrm{Al}_{2} \mathrm{O}_{3}\end{array}$ & $\begin{array}{ll} & \text { Fritte } \mathrm{G}_{4} \\
0,07 \mathrm{CaO} & 3,84 \mathrm{SiO}_{2} \\
0,25 \mathrm{~K}_{2} \mathrm{O} & 0,84 \mathrm{~B}_{2} \mathrm{O}_{3} \\
0,02 \mathrm{Na}_{2} \mathrm{O} & 0,25 \mathrm{Al}_{2} \mathrm{O}_{3} \\
0,66 \mathrm{PbO} & \\
1,00 & \\
\end{array}$ & & $\begin{array}{c}10,0 \mathrm{CaO} \\
23,5 \mathrm{PbO} \\
5,2 \mathrm{~K}_{2} \mathrm{O} \\
0,2 \mathrm{Na}_{2} \mathrm{O}\end{array}$ & $\begin{array}{l}14,5 \mathrm{SiO}_{2} \\
9,3 \mathrm{~B}_{2} \mathrm{O}_{3} \\
7,3 \mathrm{Al}_{2} \mathrm{O}_{3}\end{array}$ \\
\hline$G_{5}$ & $\begin{array}{l}70,8 \% \text { Fritte } G_{4} \\
15,3 \% \text { Kreide } \\
7,4 \% \text { Feldspat } \\
3,6 \% \text { Kaolin } \\
2,9 \% \text { Mennige }\end{array}$ & $\begin{array}{l}0,51 \mathrm{CaO} \\
0,01 \mathrm{Na}_{2} \mathrm{O} \\
0,15 \mathrm{~K}_{2} \mathrm{O} \\
0,33 \mathrm{PbO} \\
1,00\end{array}$ & $\begin{array}{l}2,08 \mathrm{SiO}_{2} \\
0,38 \mathrm{~B}_{2} \mathrm{O}_{3} \\
0,20 \mathrm{Al}_{2} \mathrm{O}_{3}\end{array}$ & $\begin{array}{l}\text { Fritte } 4 \\
\text { siehe oben bei } G_{4}\end{array}$ & & $\begin{array}{r}9,8 \mathrm{CaO} \\
25,5 \mathrm{PbO} \\
5,1 \mathrm{~K}_{2} \mathrm{O} \\
0,2 \mathrm{Na}_{2} \mathrm{O} \\
\end{array}$ & $\begin{array}{l}43,0 \mathrm{SiO} \\
9,2 \mathrm{~B}_{2} \mathrm{O}_{3} \\
7,1 \mathrm{Al}_{2}\left(\mathrm{j}_{3}\right.\end{array}$ \\
\hline$W_{16}$ & 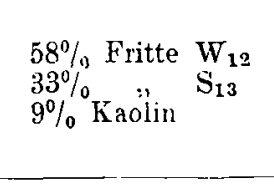 & $\begin{array}{l}0,29 \mathrm{CaO} \\
0,25 \mathrm{Na}_{2} \mathrm{O} \\
0,16 \mathrm{~K}_{2} \mathrm{O} \\
0,30 \mathrm{PbO} \\
\frac{1,00}{}\end{array}$ & $\begin{array}{l}2,64 \mathrm{SiO}_{2} \\
0,43 \mathrm{~B}_{2} \mathrm{O}_{3} \\
0,26 \mathrm{Al}_{2} \mathrm{O}_{3}\end{array}$ & $\begin{array}{ll}\text { Fritte } & \mathrm{W}_{12} \\
0,30 \mathrm{CaO} & 2,30 \mathrm{SiO}_{2} \\
0,10 \mathrm{~K}_{2} \mathrm{O} & 0,35 \mathrm{~B}_{\mathbf{2}} \mathrm{O}_{3} \\
0,60 \mathrm{PbO} & 0,10 \mathrm{Al}_{2} \mathrm{O}_{3} \\
1,00 & \end{array}$ & \begin{tabular}{ll}
\multicolumn{3}{c}{ Fritte $\mathrm{S}_{13}$} \\
$0,29 \mathrm{CaO}$ & $2,57 \mathrm{SiO}_{2}$ \\
$0,50 \mathrm{Na}_{\mathbf{2}} \mathrm{O}$ & $0,50 \mathrm{~B}_{2} \mathrm{O}_{3}$ \\
$0,21 \mathrm{~K}_{\mathbf{2}} \mathrm{O}$ & $0,22 \mathrm{Al}_{2} \mathrm{O}_{3}$ \\
1,00 &
\end{tabular} & $\begin{array}{c}8,7 \mathrm{CaO} \\
19,8 \mathrm{PbO}^{4} \\
4,6 \mathrm{Na}_{2} \mathrm{O} \\
4,5 \mathrm{~K}_{2} \mathrm{O}\end{array}$ & $\begin{array}{l}46,2 \mathrm{SiO}_{2} \\
8,8 \mathrm{~B}_{2} \mathrm{O}_{3} \\
7,6 \mathrm{Al}_{2} \mathrm{O}_{3}\end{array}$ \\
\hline$W_{2 i}$ & $\begin{aligned} 92,0 \% & \text { Fritte } \mathrm{W}_{23} \\
8,0 \% & \text { Kaolin }\end{aligned}$ & $\begin{array}{l}0,30 \mathrm{CaO} \\
0,25 \mathrm{Na}_{2} \mathrm{O} \\
0,15 \mathrm{~K}_{2} \mathrm{O} \\
\frac{0,30}{1,00} \mathrm{PbO}\end{array}$ & $\begin{array}{l}2,50 \mathrm{SiO}_{2} \\
0,40 \mathrm{~B}_{2} \mathrm{O}_{3} \\
0,25 \mathrm{Al}_{2} \mathrm{O}_{3}\end{array}$ & $\begin{array}{ll}\quad \text { Fritte } & \mathrm{W}_{23} \\
0,30 \mathrm{CaO} & 2,30 \mathrm{SiO}_{2} \\
0,25 \mathrm{Na}_{2} \mathrm{O} & 0,40 \mathrm{~B}_{2} \mathrm{O}_{3} \\
0,15 \mathrm{~K}_{2} \mathrm{O} & 0,15 \mathrm{Al}_{2} \mathrm{O}_{3} \\
0,30 \mathrm{PbO} & \\
1,00 & \end{array}$ & & $\begin{array}{c}5,3 \mathrm{CaO} \\
20,9 \mathrm{PbO} \\
5,0 \mathrm{Na}_{2} \mathrm{O} \\
4,4 \mathrm{~K}_{2} \mathrm{O}\end{array}$ & $\begin{array}{l}47,5 \mathrm{SiO}_{2} \\
8,8 \mathrm{~B}_{2} \mathrm{O}_{3} \\
8,1 \mathrm{Al}_{2} \mathrm{O}_{3}\end{array}$ \\
\hline $\mathrm{W}_{i}$ & $\begin{array}{ll}75,6^{\circ} \% & \text { Fritte } W_{7} \\
12,4^{0} \% \text { Kaolin } \\
12,0 \% \text { Quarz }\end{array}$ & $\begin{array}{l}0,48 \mathrm{CaO} \\
0,40 \mathrm{Na}_{2} \mathrm{O} \\
\frac{0,12}{1,00} \mathrm{~K}_{2} \mathrm{O}\end{array}$ & $\begin{array}{l}2,20 \mathrm{SiO}_{2} \\
0,40 \mathrm{~B}_{2} \mathrm{O}_{3} \\
0,24 \mathrm{Al}_{2} \mathrm{O}_{3}\end{array}$ & $\begin{array}{c}\text { Fritte } W_{7} \\
\text { siehe oben bei } G_{1}\end{array}$ & & $\begin{array}{c}10,8 \mathrm{CaO} \\
10,0 \mathrm{Na}_{2} \mathrm{O} \\
4,5 \mathrm{~K}_{2} \mathrm{O}\end{array}$ & $\begin{array}{c}53,5 \mathrm{SiO}_{2} \\
11,3 \mathrm{~B}_{2} \mathrm{O}_{3} \\
9,9 \mathrm{Al}_{2} \mathrm{O}_{3}\end{array}$ \\
\hline$S_{14}$ & $\begin{aligned} 83,7 \% & \text { Fritte } \mathrm{S}_{13} \\
8,9 \% & \text { Kreide } \\
7,4 \% & \text { Kaolin }\end{aligned}$ & $\begin{array}{l}0,45 \mathrm{CaO} \\
0,39 \mathrm{Na}_{2} \mathrm{O} \\
\frac{0,16}{1,00} \mathrm{~K}_{2} \mathrm{O}\end{array}$ & $\begin{array}{l}2,14 \mathrm{SiO}_{2} \\
0,39 \mathrm{~B}_{2} \mathrm{O}_{3} \\
0,24 \mathrm{Al}_{2} \mathrm{O}_{3}\end{array}$ & $\begin{array}{c}\text { Fritte } \mathrm{S}_{13} \\
\text { siehe oben bei } W_{16}\end{array}$ & & $\begin{array}{c}10,2 \mathrm{CaO} \\
9,7 \mathrm{Na}_{2} \mathrm{O} \\
6,1 \mathrm{~K}_{\mathbf{2}} \mathrm{O}\end{array}$ & $\begin{array}{l}52,7 \mathrm{SiO}_{2} \\
11,0 \mathrm{~B}_{2} \mathrm{O}_{3} \\
10,3 \mathrm{Al}_{2} \mathrm{O}_{3}\end{array}$ \\
\hline$S_{17}$ & $\begin{aligned} 92,0 \% & \text { Fritte } \mathrm{S}_{13} \\
5,0 \% & \text { Kreide } \\
3,0 \% & \text { Kaolin }\end{aligned}$ & $\begin{array}{l}0,37 \mathrm{CaO} \\
0,44 \mathrm{Na}_{2} \mathrm{O} \\
0,19 \mathrm{~K}_{2} \mathrm{O} \\
1,00\end{array}$ & $\begin{array}{l}2,30 \mathrm{SiO}_{2} \\
0,44 \mathrm{~B}_{2} \mathrm{O}_{3} \\
0,22 \mathrm{Al}_{2} \mathrm{O}_{3}\end{array}$ & $\begin{array}{c}\text { Fritte } \mathrm{S}_{13} \\
\text { siehe oben bei } \mathrm{W}_{16}\end{array}$ & & $\begin{array}{c}8,1 \mathrm{CaO} \\
10,6 \mathrm{Na}_{2} \mathrm{O} \\
6,7 \mathrm{~K}_{2} \mathrm{O}\end{array}$ & $\begin{array}{c}53,9 \mathrm{SiO}_{2} \\
12,0 \mathrm{~B}_{2} \mathrm{O}_{3} \\
8,7 \mathrm{Al}_{2} \mathrm{O}_{3}\end{array}$ \\
\hline$S_{22}$ & $\begin{aligned} 96,0 \% & \text { Fritte } \mathrm{S}_{22} \\
4,0 \% & \text { Kaolin }\end{aligned}$ & $\begin{array}{l}0,30 \mathrm{CaO} \\
0,50 \mathrm{Na}_{2} \mathrm{O} \\
\frac{0,20}{1,00} \mathrm{~K}_{2} \mathrm{O}\end{array}$ & $\begin{array}{l}2,20 \mathrm{SiO}_{2} \\
0,40 \mathrm{~B}_{2} \mathrm{O}_{3} \\
0,24 \mathrm{Al}_{2} \mathrm{O}_{3}\end{array}$ & \begin{tabular}{ll}
\multicolumn{3}{c}{ Fritte $\mathrm{S}_{\mathbf{2 2}}$} \\
$0,30 \mathrm{CaO}$ & $2,12 \mathrm{SiO}_{2}$ \\
$0,50 \mathrm{Na}_{2} \mathrm{O}$ & $0,40 \mathrm{~B}_{2} \mathrm{O}_{3}$ \\
$0,20 \mathrm{~K}_{2} \mathrm{O}$ & $0,20 \mathrm{Al}_{2} \mathrm{O}_{3}$
\end{tabular} & & $\begin{array}{c}6,7 \mathrm{CaO} \\
12,0 \mathrm{Na}_{2} \mathrm{O} \\
7,6 \mathrm{~K}_{2} \mathrm{O}\end{array}$ & $\begin{array}{r}53,3 \mathrm{SiO}_{2} \\
11,0 \mathrm{~B}_{2} \mathrm{O}_{3} \\
9,5 \mathrm{Al}_{2} \mathrm{O}_{3}\end{array}$ \\
\hline
\end{tabular}


$02-2$ eine Glasur zu schaffen, ohne aber dieses durch all$\mathrm{zu}$ hohen Gehalt an $\mathrm{PbO}$ zu erreichen. Als Ausgangsglasur ist Glasur $V$ anzusehen, die im Glattbrand zwischen SK 2 und SK 6 gebrannt befriedigende Resultate lieferte und selbst bei Temperaturen von SK 02 bereits eine blanke Glasuroberfläche lieferte, die nur bei dieser Temperatur stark eierschalig, d. h. also mit kleinsten Gasperlen durchsetzt war, die an der Oberfläche wie Nadelstiche aussehen $^{4}$ ).

Durch Verminderung der Säurestufe sollte die Möglichkeit eines niederen Glattbrandes gewonnen werden. So entstand die Glasur G 1, die aber durchaus unbrauchbare Resultate ergab. Im Rundofen gebrannt, lieferte sie bis zu SK 1 eine Glasurfläche, die schleierartig mit einer matten Schicht vollständig bedeckt war. Oberhalb dieser Temperatur begann die Auflösung dieser Schicht und zwar von der Mitte aus zunehmend, also nicht von den Plattenrändern her, wie man doch annehmen sollte, da von dort aus die Temperaturen nach dem Inneren fortschreiten. Dabei war die Lösung der Mattschicht in dünnen Glasurlagen schwieriger als in kräftigeren. Bei SK 2-SK 3 war in allgemeinen die Glasur blank bis auf einen schmalen Streifen von matter, eingetrockneter Glasur, der in der Nähe der Füllstützen oder bei rauher Kapselwandung sich verbreitert und in bezug auf seine Intensität sich bis zur Bildung faltiger Beläge und aufplatzender Bläschen steigern kann. Durch Temperaturerhöhung ist diese Randausscheidung nicht zu beseitigen, so daß überhaupt nicht die Möglichkeit vorliegt, mit dieser Glasur einwandfreie Ware zu erzielen. Dabei ist die Temperaturgrenze, innerhalb der die Mattschicht auftritt, und die Intensität der Erscheinungen nichts Feststehendes, jeder Brand bringt ein etwas anderes Resultat, nur da $\beta$ die Fehler dann umso stärker auftreten, wenn durch unvorsichtiges Feuern und ungenügendes Entschlacken der Roste zu starke Rauchperioden, besonders während des Schmelzens der Glasur, auftraten.

In Hinsicht auf die spätere Erörterung der Sulfatbildung ist bemerkenswert, daß Stücke erhalten wurden, die die Folgen eines reduzierenden Brandes bis zur Schwärzung also Bleiausscheidung zeigten und dabei trotz eines hohen Brandes eine matte Oberfläche hatten. In der Muffel gebrannt, trat ein Blankwerden der Glasurfläche bereits bei niederen Temperaturen als im Rundofen ein, die Randausscheidungen aber bestanden unvermindert fort.

Um nun gleich den großen Einfluß der Art der Unterteilung in Fritte und Glasur zu zeigen, sollen an dieser Stelle die Ergebnisse der Glasur $W_{4}$ folgen, die mit $G_{1}$ in bezug auf die molekulare Gesamtzusammensetzung nahezu vollständig übereinstimmt, deren Fritte aber sehr viel saurer ist als diejenige für die Glasur $G_{1}$ und die dementsprechend $\mathrm{CaCO}_{3}$ und die geringe Menge von nur $1,8 \%$ Mennige im Versatz hat. $W_{4}$ war bereits bei Temperaturen von SK 02 blank ausgeflossen, zeigte keine Ausscheidungen, und auch die Plattenränder blieben ungestört selbst bei Temperaturen bis zu SK 6 , bei denen allerdings die Bildung einer nadelstichigen Oberfläche sich bemerkbar machte.

Die nun versuchte Entfernung des Pbo aus dem versatz und sein Einschmelzen in die Fritte führte zu der Glasur $G_{4}$, die also molekular genau wie $W_{4}$ aussieht. Und nun trotz dieser anscheinend geringfügigen Änderung in der Art der Zugabe von nur 1,8\% Mennige tritt ein völliger Wechsel im Verhalten dieser Glasur ein. Bis zu etwa SK 2 war dieselbe nunmehr mit einer dichten Mattschicht auf der ganzen Fläche überzogen. Diese Schicht hat ein kompakteres, zusammenhängenderes Aussehen, als die mehr schleierartigen Ausscheidungen der Glasur $\mathrm{G}_{1}$. Thre Lösung vollzieht sich oberhalb SK $2-$ SK 3 im allgemeinen ebenfalls von der Mitte der Platte aus, aber doch bei weitem nicht so gleichmäßig fortschreitend und nicht so im allmählichen Ubergang wie in Falle von $G_{1}$. Vielmehr erscheinen unregelmäßig auf der matten Fläche blanke Stellen, oder auf den blanken Teilen sind noch matte Inseln und einzelne krystallartige Fleckchen vorhanden.

4) Es ist wohl zweckmäßig, die Bezeichnung ,nadelstichig “ zu wählen, da teilweise unter ,Eierschäligke ${ }^{\text {i" }}$ eine gleichzeitige Trübung der Glasur verstanden wird.
Man hat den Eindruck, daß bei $G_{1}$ die kleinen schleierartig aufliegenden Verbindungen sich nur schwer lösen,

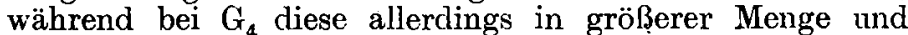
dickerer Lage vorhanden sind, sich aber, wenn einmal die Lösungstemperaturen erreicht sind, schnell lösen. Stellen dickerer Glasurlage sind noch matt, während gleich daneben die Glasurschicht blank erscheint. Da das Verschwinden der matten Decken stark von der Glasurstärke und besonders auch ron den Brennverhältnissen abhängig ist, so ist eine genaue Grenze dieser Erscheinung nicht anzugeben. Im allgemeinen ist die Fläche oberhalb SK 3 blank, und Randausscheidungen treten nicht auf. Es sind also mit dieser Glasur befriedigende Resultate zu erhalten, wenn auch nur bei Brenntemperaturen, die wesentlich höher liegen als für $W_{4}$ und den eigentlichen Schmelzpunkt stark überschreiten, was auch daraus zu schließen ist, daß selbst beim Auftreten starker Laufkanten erhebliche Teile der Glasuroberfläche noch ungeklärt sein konnten. In der Muffel gebrannt, trat die Klärung bei niederen Temperaturen ein als im Glattofen.

$\mathrm{Um}$ in dieser merkwürdigen Erscheinung sicher zu gehen, wurde nun der Glasur $\mathrm{G}_{4}$ ihrerseits wieder $\mathrm{PbO}$ im Versatz beigegeben und $z$ war $3 \%$, woraus Glasur $\mathrm{G}_{5}$ entstand, die molekular, also nur wenig von $G_{4}$ und $W_{4} a b$ weicht. $G_{5}$ lieferte genau dieselben Resultate wie $W_{4}$ war also eine betriebsichere Glasur, die oberhalb SK 01

tadellose Glasurflächen lieferte.
Hier sei eingeschaltet, daß ebenfalls für $\mathrm{G}_{1}$ eine Zugabe von $\mathrm{PbO}$ im Versatz versucht wurde und zwar mit $3 \%$. In diesem Falle zeigte sich die Zugabe nahezu wirkungslos, die Glasur verlor keineswegs ihre Mängel. Also nicht nur auf das Vorhandensein von $\mathrm{PbO}$ im Versatz kommt es an, sondern noch auf andere Faktoren.

- In dieser Hinsicht mußte man einen Einfluß des Ca0Gehaltes vermuten. So wurde zunächst $G_{1}$ im Versatz mit nahezu $5 \%$ Kreide rersetzt und die Glasur $W_{11}{ }^{5}$ ) erhalten mit dem Resultat, daß nun eine Mattschicht in gleicher Weise und bis zu gleichen Temperaturen wie bei $G_{4}$ sich bildet. Bei höheren Temperaturen verschwinden die Randausscheidungen nicht ganz.

Da nun ein gewisser Zusammenhang der auftretenden Fehler mit dem Ca0-Gehalt der Glasuren erwiesen war, so wurde nunmehr dazu übergegangen, diesen Gehalt zu vermindern bei gleichzeitiger Steigerung des Säuregehaltes, um so vor allem zunächst einmal Glasuren zu gewinnen, die ohne PbO im Versatz ein befriedigendes Resultat liefern. Dies gelang mit den Glasuren $W_{16}$ und $W_{23}$, die bei einem Glattbrand von SK 2-SK 5 Glasurflächen lieferten, die frei von Ausscheidungen auf der Fläche und an den Rändern waren. Sie waren aber beide mit steigender Temperatur zur Entwicklung von Gasperlen und zwar $W_{16}$ mehr als $\mathrm{W}_{23}$ geneigt.

Wenn im folgenden versucht werden soll, eine Erklärung für die geschilderten Ergebnisse und Resultate zu geben, so muß doch von vornherein gesagt werden, daß eine solche in Anbetracht der außerordentlich verwickelten Verhältnisse vor allem bezüglich der auftretenden chemischen Prozesse und deren Endprodukte nichts weniger als erschöpfend sein kann. Bedenkt man die Mannigfaltigkeit der Glasuren und den Mangel der Kenntnis ihrer wahren chemischen Struktur und andererseits die Veränderlichkeit der beim Brennprozeß auf sie wirkenden Faktoren in Gestalt der in jeder Temperaturzone schwer zu fassenden chemischen Zusammensetzung der Feuergase, so muß eine klare Formulierung der chemischen Vorgänge vorläufig als aussichtslos angesehen werden. Es wird also hier als Wichtigstes übrigbleiben, die Grundursachen für gewisse Erscheinungen aus einer ganzen Anzahl möglicher Ursachen herauszuschälen, um daraus die praktischen Folgerungen ziehen zu können.

Fs handelt sich bei dem geschilderten Auftreten matter Schichten ersichtlich um zwei verschiedene Erscheinungen:

1. Die matten Teile sind zum mindesten auf der Glasurfläche gleichmäßig rerteilt, wenn sie nicht dieselbe in $\mathrm{zu}-$ sammenhängender Schicht ganz bedecken. Durch Temperatursteigerung findet allmähliche Entfernung bis zum voll-

5) In der Tabelle nicht angeführt, da von $G_{1}$ nur durch Zugabe von $4,8 \% \mathrm{CaCO}_{3}$ im Versatz unterschieden. 
kommenen Blankwerden bei einer verhältnismäßig feststehenden Temperaturhöhe statt. $\mathrm{Zu}$ unterscheiden sind dabei schleierartige Trübungen und kompakte, die Glasur vollkommen bedeckende Beläge.

2. Die matten Teile treten nur an bestimmten Stellen auf, und zwar dort, wo unglasierte oder schwächer glasierte Flächen sich in der Nähe befinden, während die übrigen Teile der Glasurfläche blank erscheinen. Durch Temperatursteigerung sind diese matten Glasurflächen nicht zu beseitigen, im Gegenteil, mit höherer Temperatur verbreitern sich dieselben, und gleichzeitig erfaßt der Vorgang des Erblindens tiefere Glasurschichten. Die Oberfläche wird faltig, manchmal blasig eingetrocknet.

Wie bereits angedeutet, scheint die Bildung von Ausscheidungen auf der Glasur mit dem Gehalt an CaO in Verbindung zu stehen. Betrachtet man die prozentuale $\mathrm{Zu}$ sammensetzung der Glasuren, so zeigt sich, daß diejenigen Glasuren, die einen höheren Gehalt an $\mathrm{Ca0}$ als $10 \%$ aufweisen, die erörterten Schwierigkeiten aufweisen, besonders wenn der Gehalt an $\mathrm{SiO}_{2}$ nur etwa $45 \%$ beträgt. Da die $\mathrm{Ca}_{4}$ Silicate in der Säurestufe, wie sie hier in Betracht kommen, schwerer schmelzbar sind, als die Alkalisilicate, und vor allem auch die Vollendung der Reaktionen zwischen $\mathrm{SiO}_{2}$ und $\mathrm{CaO}$ bei höheren Temperaturen stattfindet ${ }^{6}$ ), so ist die Schwierigkeit der Auflösung von $\mathrm{CaO}$ im Versatz verständlich, ob es nun darin als freies $\mathrm{CaO}$ oder noch an Kohlensäure oder an eine andere Säure gebunden vorhanden sei, selbst wenn es sich um säurereiche Fritten handelt. Aus diesem Grund könnte man auch annehmen, daß beim Einschmelzen der Fritten für Glasur $\mathrm{G}_{1}$, die beide bei niedriger Säuerungsstufe einen hohen Gehalt an $\mathrm{CaO}$ haben, das Flüssigwerden also das Ende des Frittevorganges - eintritt, ehe alles $\mathrm{CaO}$ in Silicat verwandelt ist und in der Schwebe befindlich mitgeführt würde, so daß es späterhin beim Glasurbrand noch voliständig bis zur Klärung gelöst werden muß.

Es war zu erwarten, daß durch Einschmelzen der Glasuren in dickeren Schichten, als denjenigen des dünnen Glasurauftrages auf den Scherben, der Einblick in diese Vorgänge ein besserer sei. Dadurch mußte es sich zeigen, ob es sich um Vorgänge innerhalb der Glasur handelt, die dann in der ganzen Schicht sichtbar sein müssen. Silicatgemische können statt glasig zu erstarren, ein krystallinisches oder getrübtes Gefüge aufweisen, wie das ja bei den meisten erstarrten Schmelzprodukten der Fall ist. Derartiges müßte sich in der ganzen Schicht zeigen, ebenso wie die unvollendete Auflösung gewisser Glasurbestandteile die ganze Schicht trüben müßte. Man konnte so ein Urteil darüber gewinnen, ob es sich um Yorgänge lediglich innerhalb der Glasur handelt, oder um solche, die aus der Wechselwirkung der großen Berührungsfläche des Glasurauftrages mit dem Scherben und der Ofenatmosphäre zu erklären sind. Demzufolge wurden sämtliche Glasuren als fein gemahlenes getrocknetes Pulver in Gefäße gefüllt und im Glattbrand zu Blöcken erschmolzen.

Es zeigt sich, daß $G_{1}$ bei etwas niederer Temperatur zu einem ganz klaren Glas schmilzt als dem Klärungspunkt der Glasurschicht, also ungefähr SK 1, entspricht. Dieser Glasblock behält auch bei höheren Temperaturen sein klares blankes Aussehen. Es ist also nichts wiederzufinden von den Störungen des Erblindens der Glasurränder usw.

Dagegen liefert $G_{4}$ bei SK 1 einen getrübten, teilweise an der Oberfläche blasigen Block, ein Beweis also für lebhafte Reaktionen, die bei SK 3, wie die entsprechenden Proben zeigen, so weit vorgeschritten sind, daß die Blasenbildung beendet ist, und die Klärung des Glases zunimmt, wobei aber immer sowohl an einigen Stellen der Oberfläche, wie auch im Innern des Blocks Ungelöstes in Form von Trübungen vorhanden ist. An der Berührungsfläche von Scherben und Glasur ist der Prozeß zuletzt beendet. Bei SK 5 ist dann die Klärung vollkommen. Dieses Verhalten stimmt also mit demjenigen der Glasurschicht überein.

Und ebenso ist das mit den übrigen Glasuren der Fall, wobei es besonders bemerkenswert ist, daß die Glasur V bei niederen Temperaturen als $G_{1}$ und $G_{1}$ klar und glasig erhalten wird, daß aber der Block von kleinen Bläschen er-

6) Sprechsaal 43, 324-325 [1910] füllt erscheint, die die Durchsichtigkeit fast aufheben, in Ubereinstimmung mit dem Befund, daß diese Glasur als Glasurschicht erschmolzen schon bei Temperaturen von SK 02 bis SK 01 Glasurschichten ohne Beläge ergibt, deren Oberfläche aber stark nadelstichigartig erscheint. Bei SK 6 vermindert sich diese Erscheinung zwar, ohne aber vollstädig zu verschwinden. Dasselbe zeigen die bei SK 6 erschmolzenen Blöcke dieser Glasur.

Ein ganz ähnliches Aussehen liefern die Blöcke der Glasuren $W_{16}$ und $W_{23}$, nur ist die Bläschenbildung geringer. Frühzeitiges klares Erschmelzen des Blockes zeigten die Glasuren $W_{4}$ und $G_{5}$, bei denen auch die Bläschenbildung nahezu verschwunden war, ebenfalls in Ưbereinstimmung mit ihrem Verhalten als Glasurschicht, die innerhalb der untersuchten Temperaturen vollkommen glatt und blank waren.

Diese Resultate zeigen also nur für die Glasur $C_{x}$ ein Verhalten, das nicht mit demjenigen der Glasurschicht in Ubbereinstimmung steht. Das frühzeitigere Klarwerden der als Block geschmolzenen Glasur würde nicht eintreten, wenn es sich lediglich um die Auflösung von ungelöstem $\mathrm{CaO}$ handelte, wie ja auch schon das Aussehen der nicht genügend hochgebrannten Glasurschicht $G_{1}$ ein wesentlich anderes ist, als das von $\mathbf{G}_{\mathbf{4}}$.

Es müssen also hier zur Erklärung der Vorgänge noch andere Faktoren herangezogen werden, und da weist das am Einschmelzen in Block gewonnene Resultat daraufhin, die von außen kommenden Einwirkungen der Feuergase oder des Scherbens zu berücksichtigen.

Von diesem Gesichtspunkt aus erscheint es vor allem wichtig, den Einfluß der Sulfatbildung in den Glasuren zu betrachten, die seit Seg e $\mathrm{S}^{7}$ ) grundlegenden Betrachtungen immer wieder als Grund für die Störungen der Glasuroberfläche in Form von Erblinden der Oberfläche und Ausscheidungen auf derselben herangezogen werden, um so mehr, als die Fehler der Glasuren $\mathrm{G}_{1}$ zuerst hierauf zurückgeführt, und die bereits geschilderten Anderungen an dieser Glasur in diesem Hinblick vorgenommen wurden.

Geht man bezüglich der Glasur $G_{1}$ von der Ansicht aus, daß die vor der vollständigen Klärung der Glasur auftretenden Trübungen durch Sulfate bedingt seien, so gäbe allerdings die Tatsache, daß die beiden Fritten $W_{7}$ und $W_{9}$ basischer sind als die endgültige Glasur $G_{1}$, die aus beiden und dem Versatz sich bildet, dazu eine gute Erklärung. Nach den S e ge r schen Feststellungen enthält ein Disilicat $4 \%$, ein Trisilicat $2 \% \mathrm{H}_{2} \mathrm{SO}_{4}$, so da 3 also ganz allgemein für die untersuchten Glasuren, die sich innerhalb der Di- und Trisilicate bewegen, festzulegen wäre, daß bei Erhöhung der Säurestufe infolge von Auflösung des Versatzes durch die Fritte eine Ausscheidung von Sulfaten aus dem Glasfluß eintritt, wenn die Fritte selbst an diesen gesättigt war. Und umgekehrt: Daß selbst für diesen letzteren Fall alles Sulfat gelöst bleibt, wenn der Versatz die Fritte basischer werden läßt. Die Erfahrungen an der Glasur $\mathrm{G}_{4}$, deren Säurestufe in ausgesprochenem Maße durch den Versatz vermindert wird, lehren, daß es sich keinesfalls in den vorliegenden Fällen um die Abscheidung von Sulfaten handeln kann, was ebenfalls die Glasuren $W_{16}$ und $W_{23}$ bestätigen, deren Säuerungsstufen durch den Versatz erhöht werden.

Da nun aber gerade neuerdings $P$ u k a l l ${ }^{8}$ ) und $D$ or $f$ $n$ e $\mathbf{r}^{9}$ ) bei ihren Untersuchungen über bleifreie Glasuren die Ausscheidung von Sulfaten als ein Grund von Störungen der Glasuroberfläche annehmen, so schien es immerhin erforderlich, dieser Frage durch quantitative Bestimmungen von Schwefelsäure auf den Grund zu gehen, wozu die als Blöcke erschmolzenen Glasuren eine Möglichkeit boten, während das für die dünne Schicht eines Glasurauftrages auf den Scherben größere Schwierigkeiten bereitet.

Leider hat $\mathbf{S}$ e g e $\mathbf{r}$ selbst nichts über den eigentlichen quantitativen Nachweis mitgeteilt. Es zeigte sich bei der Ausarbeitung einer Bestimmungsmethode, daß durch Aufschließen der fein gepulverten Glasur mit Carbonatgemisch in üblicher Weise, Abscheidung der Kieselsäure und Aus-

i) Se ger s Gesammelte Sohriften. S. 527.

8) Sprechsaal 47, 77 [1914]. Sprechsaal 47, 390 [1914]. 
fällen der Sulfate mit $\mathrm{BaCl}_{2}$ im Filtrat übereinstimmende Resultate nicht zu erzielen waren, da $\mathrm{CaSO}_{4}$ und $\mathrm{PbSO}_{4}$ infolge ihrer Schwerlöslichkeit zum Teil mit der Kieselsäure abgeschieden werden, was durch einen erheblichen Rück stand beim Abrauchen der abgeschiedenen und geglühten Kieselsäure bekräftigt wurde. Auch durch Kochen der gepulverten Glasur mit viel Wasser oder mit $10 \%$ iger Sodalösung wurde Befriedigendes nicht erzielt, wobei allerdings die Ergebnisse der letzteren Methode sich sehr demjenigen der als einwandfrei gefundenen Bestimmung näherte. Diese bestand darin, auf eine vollkommene Abscheidung der $\mathrm{SiO}_{2}$ aus dem Aufschluß der Glasur mit Carbonatgemisch zu verzichten, vielmehr denselben mit viel Wasser zu behandeln, (700 ccm bei ca. 0,5 g Substanz) und mit $\mathrm{HCl}$ sauer zu machen. Infolge der starken Verdünnung blieb in manchen Fällen die Kieselsäure gelöst, in anderen entstand eine teilweise Ausfällung, die abfiltriert wurde, um im Fil trat dann die Sulfate in $\mathrm{BaSO}_{4}$ überzuführen. Dabei könnten als schwerlöslich immerhin Ba-Borat und -Silicat mit ausfallen; beim Abrauchen des abgeschiedenen $\mathrm{BaSO}_{4}$ mit $\mathrm{HF}$ trat aber nur ein geringer Gewichtsverlust ein, so da $B$ dieses Bestimmungsverfahren zum mindestens für den vorliegenden Zweck ausreichte.

Es wurde nun für die Glasur $\mathrm{G}_{1}$, bei SK 5 im Glattofen gebrannt, ein Gehalt an $1,54 \% \mathrm{H}_{2} \mathrm{SO}_{4}$ und bei SK 03 in der Muffel gebrannt ein solcher von $1,16 \mathrm{H}_{2} \mathrm{SO}_{4}$ gefunden. Die ungebrannten, zum Glasieren fertigen Glasuren $\mathrm{G}_{4}$ und $\mathrm{W}_{4}$ enthielten 1,48 und $1,84 \%$ und die Fritte $1550,84 \% \mathrm{H}_{2} \mathrm{SO}_{4}$. Es wurde dann aber mit der bleifreien Glasur $W_{7}$ beim Brande im Ringofen ein Schmelzkuchen erhalten, der mit einer ablösbaren Kruste von Salzen bedeckt war, die sich bei der Analyse als zu $52 \%$ aus $\mathrm{Na}_{2} \mathrm{SO}_{4}$ und zu $48 \%$ aus $\mathrm{CaSO}_{4}$ bestehend erwiesen. Der Schmelzkuchen selbst war im übrigen klar. Er muß, da er Sulfate abgeschieden hat, mit diesen gesättigt sein, und die Analyse ergab einen Gehalt von 3,5\% $\mathrm{H}_{2} \mathrm{SO}_{4}$, was also mit den Angaben $\mathrm{Segers}$ gut übereinstimmt.

Was zeigen die bemerkenswerten Analysenresultate? Das sowohl durch die Materialien selbst als auch durch den Prozeß des Frittens nicht solche Sulfatmengen eingeführt wer den, daß die Glasur aus sich mit diesen gesättigt ist. $\mathrm{Zu}$ nächst beweist das der Gehalt an Sulfaten in der bei SK 5 gebrannten Glasur $G_{1}$, bei welcher ja gerade die Störungen der Glasuroberfläche erheblich auftreten, und in schlagenderer Weise aber noch der Gehalt dieser Glasur, als sie in der Muffel gebrannt wurde. Der erhaltene Schmelzkuchen war blasig und getrübt, man hätte also auf eine erfolgte Ausscheidung von Sulfate, die infolge der mehr neutralen $\mathrm{Na}$ tur der Muffelatmosphäre bisher nicht zersetzt werden konnten, und ihre nunmehrige Zerstörung durch Reaktionen innerhalb der Glasur unter blasenbildendem Entweichen von $\mathrm{SO}_{3}$ schließen können. Trotzdem ein noch geringerer $\mathrm{Ge}$ halt an Sulfaten, so daß für diese Vorgänge in der Glasur ganz andere Gründe vorliegen müssen. Und nun endlich, trotz des nur geringfügigen Vorhandenseins von Sulfaten in der ungeschmolzenen Glasur, bei $W_{7}$ eine Utbersättigung von Sulfaten. Daß dieses beim Brand im Ringofen - also bei sehr langer Dauer des Brandes und sauerstoffreicher Feuerführung - eintritt, weist nun unbedingt darauf hin, daß durch den Brand selbst sich die Bildung von Sulfaten in der Glasur vollziehen kann, worauf ja auch schon $\mathrm{S}$ e g e r hingewiesen hat, und wofür hier der Nachweis erbracht ist. Berücksichtigt man, daß für $W_{7}^{\prime}$ dieses sogar innerhalb des Schmelzkuchens eingetreten ist, $\mathrm{da} B$ aber im Falle einer Glasurschicht durch die großen Berührungsflächen mit dem Scherben und den Ofengasen ganz andere Bedingungen vorliegen, so wird es klar, daß aus den für die Schmelzkuchen erhaltenen Resultaten nicht ohne weiteres geschlossen werden kann, daß auch in denselben, als Schicht aufgeschmolzenen Glasuren gleich geringe Mengen Sulfate vorhanden sind. Trotzdem kann es sich aber bei den geschilderten Ausscheidungen nicht um unzersetzte Sulfate handeln, denn eine Sulfatschicht müßte, wie es ja bei W, der Fall war, mechanisch ablösbar oder doch zum mindestens durch Wasser auflösbar sein. Das ist aber bei den Erscheinungen der Glasurschichten von $G_{2}$ und $G_{4}$ niemals der Fall gewesen. Die vorliegende Art des Glattbrandes mit abwechselnden
Perioden von rauchiger und sauerstoffreicher Feuerführung ist gerade das wirksamste Mittel der Zerstörung von Sulfaten. Wenn sie also an der Oberfläche ausgeschieden waren, so mußten sie allerdings der baldigen Zersetzung anheim fallen, wobei aber - was bisher von anderer Seite immer übersehen wurde - die betreffende Base als Rückstand bleibt und im weiteren Verlauf des Brandes von den Glasurschichten gelöst werden muß. Handelt es sich um $\mathrm{CaSO}_{4}$ - und für $\mathrm{W}_{7}$ ist das ja zu $50 \%$ in der Ausscheidung gefunden worden - und ist an sich die Glasur zur Aufnahme von $\mathrm{CaO}$ wenig geneigt, so kann die Lösung des $\mathrm{CaO}$ durch die Glasur Schwierigkeiten bereiten und infolgedessen zur Bildung einer Schicht von ungelöstem $\mathrm{CaO}$ auf der Glasuroberfläche bei frühzeitiger Unterbrechung der Brennprozesse führen. Der Ring beginnt sich zu schließen: So liegen Dinge anscheinend für die Glasur $G_{4}$. Die Bildung von Sulfaten während des Brandes ist erwiesen. Dabei denkt man zuerst an ein Niederschlagen von $\mathrm{H}_{2} \mathrm{SO}_{4}$-haltigem Wasser zu Beginn des Brennprozesses auf Glasur und Scherben und an ein Vollsaugen des letzteren. Im weiteren Verlauf tritt Verdunstung des Wassers an der Oberfläche und zwar in Anbetracht der Füllweise von Wandplatten, gerade an der glasierten Oberfläche und vor allem an den Seitenkanten und durch Nachdringen aus den tieferen Schichten eine Konzentration der Schwefelsäure ein, so daß man es in gewissen Stadien des Brennprozesses mit einer intensiven Einwirkung hochprozentiger Schwefelsäure bei hohen Temperaturen zu tun hat, die im Falle des Vorhandenseins von $\mathrm{CaCO}_{3}$ im Versatz dieses ganz in $\mathrm{CaSO}_{4}$ umsetzen wird, die aber auch an der Fritte gerade infolge ihrer feinsten Zerteilung sicherlich nicht spurlos vorübergeht, sondern $\mathrm{zu}$ einer teilweisen Zersetzung der Silicate und vor allem Borate unter Bildungen von Sulfaten mit den vorhandenen Basen führen muß. Es kommt nun alles darauf an, ob diese Sulfate durch ieduzierende Flammenzuführung zerstört werden, bevor die Glasur zum Schmelzen gelangt oder nicht. Ist das nicht der Fall, so findet die schmelzende Glasur wahrscheinlich so viel Sulfat vor, daß sie sich nicht nur mit diesem sättigt, sondern noch Sulfat im Utberschuß bleibt, so daß demgegenüber die etwa durch den Versatz bedingte Sulfatabscheidung ohne Belang ist, und man es in jedern Falle, in welcher Weise auch die Unterteilung der Glasuren in Fritte und Versatz bewirkt sei, mit einem als Ausscheidung auftretenden Sulfatüberschuß zu tun hat der wenigstens für die Erdalkali und Alkalisulfate sich erst bei SK 9, (nach $\mathrm{Puk}$ a $1 \mathbf{l}^{\mathbf{1 0}}$ )) zersetzt. Aus den mit den verschiedenen Glasuren gewonnenen Ergebnissen trifft dieses für die vorliegenden Verhältnisse keinesfalls zu, da ja auch der teilweise reduzierende Brand ein dauerndes Bestehen der Sulfate ausschließt.

Vielmehr muß angenommen werden, daß, wenn auch nicht alle Sulfate bei der beginnenden Schmelze zerstört sind, dieses doch mit steigender Temperatur und vor der Beendigung des Brandes vollständig geschieht, soweit nicht inzwischen die Lösung eingetreten ist, so daß man es nur noch mit den Wirkungen der Sulfatbildung in den früheren Perioden des Brandes zu tun hat.

In dieser Hinsicht ist nun für $G_{1}$ anzunehmen, daß ein Teil der Ca-Silicate oder -Borate zersetzt wurde, und die Schwierigkeiten bei der Klärung dieser Glasur in der erforderlichen Wiederauflösung des bei der Zersetzung der Sulfate zurückbleibenden $\mathrm{CaO}$ bestehen, vor allem in Anbetracht dessen, da $B$ diese Glasur wenig sauer ist. \& Bei den wesentlich saueren Glasuren $W_{16}$ und $W_{23}$ vollzieht sich diese Auflösung viel leichter, und zwar um so leichter, je geringer der Gesamtgehalt an $\mathrm{CaO}$ ist. Wie schon erwähnt, wird bei $\mathrm{G}_{4}$ das $\mathrm{CaCO}_{3}$ des Versatzes in $\mathrm{CaSO}_{4}$ umgesetzt, wobei es sich um beträchtliche Mengen handelt. Das ziemlich plötzliche Verschwinden des kräftigen Belages bei SK 3 läßt darauf schließen, daß die Zerstörung solcher Mengen $\mathrm{CaSO}_{4}$ erst bei höherer Temperatur erfolgt, so daß sie einer Einwirkung durch die schmelzende Fritte länger widerstehen, als wenn es sich nur um $\mathrm{CaO}$ handelte. Der Zusatz von nur 3\% Mennige wird in Verbindung mit dem Kaolin des Versatzes mit diesen letzteren für sich silicatbildend wir-

10) Wie oben S. 425 . 
ken und einen frühzeitigeren Angriff des $\mathrm{CaSO}_{4}$ und Lösung des $\mathrm{CaO}$ herbeiführen, so daß die merkwürỏige und intensive Verbesserung in Hinsicht auf das Blankwerden der Glasurschicht durch diesen geringen Zusatz klar wird.

Schließlich bleibt die Bildung matter Randzonen nach der erfolgten Klärung der Glasur $G_{1}$ zu erörtern. Das Charakteristische dieser Erscheinung ist, daß sie eben an den Rändern, wo also doch infolge der Nähe der Kapselwanclung die höchsten Temperaturen herrschen, auftreten, und in Ubereinstimmung damit gerade an Stellen höchster Temperatur im Ofen, an den Feuereintrittsstellen, am weitesten auf die Platte ragen und sich bis zur Falten- und Blasenbildung steigern. Es liegt also nahe, Verdampfungserscheinungen anzunehmen, die einmal durch Temperaturerhöhung, dann aber auch durch das Vorhandensein poröser oder unglasierter Teile gesteigert werden, also durch Füllstützen und in erster Linie durch die von Glasur befreiten Kanten der Platte selbst.

Man könnte dabei zunächst an $\mathrm{PbO}$ denken; die Tatsache aber, daß die bleifreie Glasur $\mathrm{S}_{17}$ ebenfalls Randausscheidungen zeigte, ließ doch einen $Z$ weifel daran aufkommen. Zur Klärung dieser ganzen Frage wurden zunächst die glasierten Platten so gefüllt, daß jedesmal die Glasurseite einer unglasierten Seite im Brand dicht gegenüber stand, und das Ergebnis war eine Bestätigung dafür, daß Destillationen aus der Glasur zu porösen Stellen der Grund für die Erscheinung sein mußte, indem nunmehr die ganze Glasurfläche der Glasur $G_{1}$ mit einem Schleier von Ausscheidungen bedeckt war. Dagegen hatte diese Füllweise auf $G_{5}$, die auch bei normaler Füllweise ohne Randausscheidungen blieb, keinen Einfluß, während bei $G_{4}$ die Klärung in bezug auf die erforderliche Temperaturhöhe verzögert wurde. Für $W_{\text {Is }}$ und $W_{23}$ zeigte sich ein Einflu $\beta$ nur insofern, als in erhöhtem Maße - und bei SK 6 stärker als bei SK 3-die Glasurschicht mit kleinsten Bläschen durchsetzt war. Zugleich war es interessant, zu beobachten, daß die rauhen Flächen, die $G_{1}$ gegenübergestanden hatten, einen leichten Glanz aufwiesen, während diejenige, die $G_{5}$ gegenüberstand, fast unvermindert stark saugfähig blieb und doch gerade für die letztere bei dem Vorhandensein von freiem $\mathrm{PbO}$ im Versatz bei etwaiger Verdampfung das Gegenteil der Fall sein mußte. Schließlich wurde nun den Glasuren $G_{1}$ und $G_{5}$ eine mit Mennige und eine mit Borsäure bestrichene, sonst rauhe Platte im Brande gegenübergestellt: Und wieder bei $G_{x_{5}}$ keine Einwirkung, dagegen bei $G_{1}$ und wie auch bei $\mathrm{G}_{1}+3 \%$ Mennige unverminderter Schleierbelag, aber gegenüber der Borsäurefläche nunmehr auch eine blanke Glasurschicht. Es ist somit erwiesen, daß eine Destillation von Borsäure in Glasuren im Brande stattfinden kann und zwar in bedeutendem Maße, wenn diese Borsäure von porösen Teilen aufgenommen werden kann.

Wenn es sich nun tatsächlich um Borsäureverdampfung in der Temperaturzone des Glattbrandes handelt, so muß es außerordentlich merkwürdig erscheinen, daß trotz gleicher stöchiometrischer Zusammensetzung $G_{1}$ die Folgen dieses Verdampfens in Form der Randabscheidungen zeigt, $\mathbf{G}_{5}$ aber nicht. Man müßte annehmen, daß, von einer bestimmten Temperatur beginnend, ein Verdampfen der Borsäure eintreten und für Glasuren gleicher Zusammensetzung im gleichen Brand die gleiche Wirkung haben müßte. Das aber ist keineswegs der Fall : $G_{1}$ wird durch rauhe Flächen schle erartig getrübt, $G_{5}$ bleibt vollkommen blank. Dieser Widerspruch ist nur dadurch zu lösen, daß für $G_{1}$ die Neigung zur Borsäureabgabe größer ist, als für $G_{5}$, und das ist dann möglich, wenn etwa $\mathrm{B}_{2} \mathrm{O}_{3}$ ungebunden vorliegt, und dieses in niederen Temperaturzonen verdampft. Erinnert man sich nun der vorher nachgewiesenen Einwirkung von $\mathrm{H}_{2} \mathrm{SO}_{4}$ auf die noch nicht geschmolzene Glasur, so ist es ohne weiteres verständlich, daß die Borate der Fritte zersetzt werden unter Isolierung von Borsäure, die dann verdampft, ehe die unangegriffenen Glasurteile schmelzen, und eine Rückbildung der Borate erfolgen kann. Betrachtet man die Segerformel der Glasur $G_{1}$, so erkennt man, daß durch eine Entfernung der Borsäure die Formel sich stark derjenigen borsäurefreier Mattglasuren nähert ${ }^{11}$ ), daß man es

11) B e r d e l, Anleitung ziz keramischen Versuchen S. 22. also bei den schleierartigen Schichten auf der Glasur mit Bildungen derartiger Mattglasurteile zu tun hat, die erst wieder von der eigentlichen Glasur gelöst werden müssen, und weniger um die Auflösung des durch $\mathrm{H}_{2} \mathrm{SO}_{4}$ isolierten $\mathrm{CaO}$, da ja in diesem Fall die Zugabe von $\mathrm{PbO}$ zum Versatz ebenso wie bei $\mathrm{G}_{4}$ diese Lösung stark beschleunigen müßte, was nicht der Fall war.

Bei einem weiteren Entzug von Borsäure aus den noch verbliebenen oder wiedergebildeten Boraten, wie es an den Plattenrändern der Fall ist, ist die Glasur schließlich nicht mehr imstande, glasig zu werden, sondern an diesen Stellen bleibt nur die Bildung einer Mattglasur möglich. Im Falle der Glasuren $G_{4}$ und $G_{5}$ aber werden die Borate vor der Zersetzung durch das im Versatz vorhandene $\mathrm{CaCO}_{3}$, das $\mathrm{H}_{2} \mathrm{SO}_{4}$ bindet, geschützt, und die Verdampfung von Borsäure aus dem Borate tritt nun also in weit geringerem Umfange bei höheren Temperaturen ein, so übt dieser Verlust keine mattwerdenden Wirkungen aus, weil eben vorher keine Borsäureverluste eingetreten waren; es kann höchstens die Aufnahme der $\mathrm{CaO}$ durch die Glasur verzögert werden, wie das bei $G_{4}$, mit rauher Fläche gebrannt, beobachtet wurde, die aber im ganzen wie $G_{5}$ eine blanke Oberfläche aufwies.

Eine hervorragende Stütze dieser Erklärung lieferte das Ergebnis eines zweimaligen Brandes einer mit $G_{5}$ glasierten und beidemal einer rauhen Platte gegenüber gefüllten Platte: Nach dem ersten war die Glasurfläche blank wie immer, nach der zweiten aber ebenso schleierartig bedeckt wie $G_{1}$, weil eben im zweiten Brande das schützende $\mathrm{CaCO}_{3}$ fehlte, so einen ganz hervorstehenden Beweis für den Einfluß des Versatzes bildend. Selbst normal gefüllt, liefert $\mathrm{G}_{5}$ bei einem zweiten Brand einen schmalen blinden Rand, und auch $W_{16}$ wider stand einem zweiten Brand mit rauher Fläche nicht, indem sie nach einem solchen ebenfalls schleierartig bedeckt war. Sonst äußerte sich die Wirkung der rauhen Fläche bei $W_{10}$ und $W_{23}$ im ersten Brand im verstärkten Auftreten nadelstichiger Oberflächen, eine Erscheinung, die schon häufiger bei den säurereicheren Glasuren (etwa von 2,5 Äquivalent $\mathrm{SiO}_{2}$ an) angetroffen wurde. Auch dabei kann es sich woh. um nichts anderes handeln, als um verdampfende Borsäure weil rauhe Flächen auch diese Erscheinung verstärken. In folge ihres höheren Säuregehaltes weichen diese Glasuren mehr von dem Typus der Mattglasuren ab, und die Wiederauflösung von Basen kann leichter erfolgen.

Es wird nun verständlich, daß die besprochenen Erscheinungen nicht etwas für eine bestimmte Temperatur Feststehendes darstellen, daß vielmehr für die gleiche Glasur das Auftreten der Fehler derartig an Umfang und in bezug auf die Brenntemperatur wechselt, daß man ihnen zunächst hoffnungslos gegenüberstehen mu $\beta$. Abgesehen von der Füllweise, von der Art der Kapselwand wird der Ausfall der Glasur davon abhängen, welche Mengen Schwefelsäure beim Brande entstehen und ihre Wirkung auf die ungeschmolzene Glasur ausüben können, und in welcher Periode des Branáes sich die Zerstörung der gebildeten Sulfate vollzieht. Es sei in dieser Beziehung berichtet, daß die Glasur $\mathrm{G}_{2}{ }^{12}$ ) im Ringofen erst bei $K 4$ von ihrer matten Schicht befreit wurde, während das im Rundofen bereits bei $\mathrm{K} 1-\mathrm{K} 2$ geschah. $\mathrm{G}_{1}$ ist, in der Muffel gebrannt, bei SK 04 annähernd so blank wie bei K 1 im Rundofen gebrannt. Andererseits aber kann nicht angenommen werčen, daß in der Muffel die Glasuren vor der Einwirkung des Schwefels der Kohle geschützt waren, indem die Glasur Abscheidungen und Beläge ebenfalls aufwiesen, wenn auch nicht im gleichen Umfange wie im Rundofen, und vor allem aber auch Randausscheidungen unverändert auftreten.

Bleifreie Glasuren wurden ebenfalls in die Untersuchung einbezogen; sie sind in der Tabelle als $W_{7}, S_{14}, S_{17}$ und $S_{22}$ angeführt. Trotzdem ihre Formel zum Tel genau den von $\mathrm{D}$ orf $\mathrm{ner}^{13}$ ) angeführten entsprachen, die nach dessen Angabe betriebsfertige Glasuren für SK 010-SK 07 liefern, wurden doch mit ihnen im vorliegenden Fall für den Glattbrand SK 1-SK 5 keine sicheren Resultate erzielt. Bei Temperaturen von SK 1-SK 3 traten vor allem die matten

12) Sie ist nicht in der Tabelle angeführt, da sie nur unbedeutend von $G_{1}$ in Zusammensetzung und Verhalten ab.weicht.

13) Sprechsaal 4\%, 390 [1914]. 
Beläge auf, wie sie bereits an $G_{4}$ beschrieben wurden. Auch in diesen Fällen begann die Auflösung von der Mitte der Platte aus. $S_{14}$ machte in dieser Hinsicht die größten Schwierigkeiten, was auch durch den hohen Gehalt an $\mathrm{CaCO}_{3} \mathrm{im}$ Versatz verständlich ist. Bei höheren Temperaturen von SK 4 - SK 5 wurden zwar die Oberflächen im allgemeinen blank, aber in dickeren Lagen traten milchige Trübungen und nadelstichige Glasurflächen auf. In Gefäße eingeschmolzen lieferte $W_{7}$ bei SK 03 und SK 1 einen vollkommen blasigen, getrübten, $\mathrm{S}_{14}$ einen ebenfalls porzellanartig steinigen, aber blasenfreien Block. Im Ringofen bei SK $02 \mathrm{~W}_{7}$ gebrannt, wurden ganz gleiche Blöcke erhalten, die num aber Salzablagerungen an der Oberfläche zeigten, so daß es sich in diesem $\mathrm{Fall}$ wohl um $\mathrm{SO}_{3}$-Abgabe bei der Blasenbildung handeln kann. Im Glattofen dagegen waren die Glasurblöcke bei SK 3 und SK 5 klar und nur mit Glasperlen durchsetzt, auch milchige Trübungen zeigten sich dabei nicht. Die Wirkung rauher Flächen äußerte sich bei $S_{17}$ in starker Eierschäligkeit der Glasur, die sich bis zu wellig glanzlosem Aussehen steigerte.

Für $\mathrm{S}_{22}$ trat trotz Verminderung des $\mathrm{CaO}$ - und Erhöhung de: $\mathrm{SiO}_{2}$-Gehaltes wohl bezüglich aller Fehler eine Besserung ein, aber die Störungen durch Borsäureverdampfung blieben bestehen. Für die Entwicklung der Unterglasurfarben war diese Glasur ganz unbrauchbar.

Im ganzen kann festgestellt werden, daß fast mit jedem Brand die Stärke der auftretenden Mängel wechselte. Es ist zweifellos - und so erklären sich wohl auch die Abweichungen von den Dorfn erschen Ergebnissen daß infolge des Fehlens von $\mathrm{PbO}$ in erhöhtem Maße eine Abhängigkeit von dem Grad der Schwefelsäurebildung während des Brandes eintritt. Die durch die Einwirkung der Schwefelsäure auf die ungeschmolzene Glasur entstehenden Sulfate sind anscheinend schwerer zersetzbar, oder die als Rückstand verbleibenden Oxyde in der übrigbleibenden Glasur schwerer löslich, was in Erinnerung an die außerordentliche Wirksamkeit von $\mathrm{PbO}$ im Versatz bei dem gänzlichen Fehlen desselben verständlich ist. Man. kann nun rückschließend noch annehmen, daß bei Bleiglasuren durch die Schwefelsäure vor allem die Bleiborate oder Silicate zersetzt werden, daß infolgedessen die nachherige Lösung des $\mathrm{PbO}$ nach der Zersetzung der Sulfate keine Schwierigkeiten bereitet, abgesehen davon, da $\mathrm{B}$ auch $\mathrm{PbSO}_{4}$ leichter zersetzlich sein wird, als die Alkali- und Erdalkalisulfate, und daB, wie es an $G_{1}$ beobachtet wurde, im wesentlichen die Folgen des Borsäureverhustes zu überwinden sind. $\mathrm{Zu}$ diesem tritt aber bei bleifreien Glasuren die größere Schwierigkeit der Wiederauflösung der abgeschiedenen Blasen, was ja im Falle von $\mathrm{CaO}$ besondere Schwierigkeiten bereitet. Dabei wird man auf das Mittel der Schutzwirkung durch $\mathrm{CaCO}_{3}$ verzichten müssen, weil auch da die Schwierigkeiten der lösung von $\mathrm{CaO}$ auftreten und nicht durch $\mathrm{Zugabe}$ von $\mathrm{PbO}$ im Versatz gemildert wercien können. Es soll nicht unerwähnt bleiben, daß wegen der größeren Beständigkeit der gebildeten Sulfate, eher die Möglichkeit eintritt, daß die Glasur sich trotz reduzierenden Brandes an diesem sättigt, und dann natürlich auch leicht eine Abscheidung eintritt. Die Untersuchung dieser noch unvollkommen geklärten Verhältnisse wird weiter geführt.

\section{Zusammenfassung.}

Die Art der Unterteilung von Steingutglasuren in Fritte und Versatz ist namentlich für Glasuren von niederer Säurestufe von ausschlaggebender Bedeutung. Sie steht im Zusammenhang mit einer Reihe bekannter Glasurfehler, wie schleierartigen Trübungen, matten Belägen, Erblinden von Glasurrändern und nadelstichigen Oberflächenbildungen, deren Natur und Beseitigungsmöglichkeit bisher mehr oder weniger ungeklärt waren. Diese Zusammenhänge, wie auch die Wirkung der Gesamtzusammensetzung der Glasuren und der Brennweise wurden aufgefunden und eine Erklärung versucht.

Bei normalen Steingutglasuren für Glattbrandtemperaturen von SK 01-SK 6 wie sie im wesentlichen für besseres Steingut zur Verwendung gelangen, ist zu sagen, daß die Fehler im allgemeinen durch einen zu hohen Gehalt an $\mathrm{CaO}$ veranlaßt werden. Derselbe sollte bei Glasuren von mehr als 2,5 Äquivalenten $\mathrm{SiO}_{2}$ seinerseits 0,4 Äquivalente nicht übersteigen und muß erniedrigt werden in dem Maße, wie die Säurestufe sinkt.

Dabei ist zu beachten, daß eine um so schwierigere Aufnahme des $\mathrm{CaO}$ durch die Glasur in dem Maße erfolgt, wie $\mathrm{CaO}$ nicht eingefrittet, sondern im Versatz zugefügt wird.

Erblinden in der Nähe poröser Teile tritt durch Verdampfen von $\mathrm{B}_{2} \mathrm{O}_{3}$ aus der Glasur auf und zwar umsomehr, je mehr die Glasur der Einwirkung von Schwefelsäure aus den Feuergasen ausgesetzt war. Diese Abgabe verursacht bei Glasuren mit weniger als 2,5 Äquivalenten $\mathrm{SiO}_{2}$ und $0,4-0,5$ Äquivalenten $\mathrm{CaO}$ matte Ausscheidungen, vollständiges Verzehren der Glasur und aufplatzende größere Blasen, während mit steigendem $\mathrm{SiO}_{2}$ - und fallendem $\mathrm{CaO}$ Gehalt diese Gefahr verschwindet, und nur eine nadelstichigartige Glasuroberfläche eintritt.

Das Vorhandensein von $\mathrm{CaCO}_{3}$ wirkt als Schutzmittel gegen die Angriffe von $\mathrm{H}_{2} \mathrm{SO}_{4}$ auf cie Glasur und somit gegen die Borsäureverdampfung.

$\mathrm{PbO}$ im Versatz ist ein außerordentlich wirksames Mittel zur glatten Aufnahme von $\mathrm{CaO}$ auch aus dem Versatz in Glasuren, die bei Zuführung des $\mathrm{PbO}$ lediglich durch die Fritte und bei sonst gleichem Gebalt an diesem Oxyd nur bei wesentlich höherer Temperatur dazu in der Lage sind.

Auf die Borsäureverdampfung übt $\mathrm{PbO}$ im Versatz keinen Einfluß aus. Diese Gesichtspunkte liefern Richt linien :

1. Zur Vermincierung des $\mathrm{PbO}$-Gehaltes und damit zur Verbilligung von Glasuren und zur Erhöhung des Gehaltes an wohifeilem $\mathrm{CaO}$.

2. Demzufolge für die Verwendung bleifreier Glasuren, deren Betriebssicherheit gerade durch das Auftreten der geschilderten Fehler beschränkt ist.

3. Zur Einfrittung desjenigen Teiles von $\mathrm{PbO}$ der in vorhandenen Glasuren als Versatz eingeführt wurde.

4. Zur Verhinderung von Verlusten an der so ungeheuer verteuerten Borsäure.

5. Zur Verminderung nadelstichiger Oberflächen von Glasuren.

6. Zur Vermeiaung der durch Verwendung schwefelhaltiger Kohle beaingten Fehler und zur Anpassung an veränderte Brennbedingungen.

7. Zur Erzielung einwandfreier farbiger Glasuren, deren Charakter durch die oft beträchtliche Zugabe von Metalloxyden oder Ca $\mathrm{O}$-haltiger Farben stark gegenüber demjenigen der unversetzten Glasur geändert wird.

\section{Über die. Haltbarkeit feldgrauer Tuche.}

$$
\text { lou Dr. Eugen Sevil, Stutgart. }
$$

(Schlub vou S. 416.)

Da nun die verwendeten Farben und namentlich der Indigo selbst nicht die geringe Haltbarkeit des Feldgraus bedingen und darüber auch bei denjenigen Melangen, in denen der mit Indigo vorgeblaute Teil nicht mit sauren Farbstoffen nachbehandelt ist, nicht geklagt wird, liegt die Annahme nahe, da $B$ bei gleichstarker Walke der Fehler wohl in $\mathrm{de} r$ A r t und We i s e d e r F $\ddot{a} r$ b u $n$ g zu suchen sein dürfte. Die fruher geäußerte Vermutung, daß die in alkalischer Lösung gefärbte Wolle durch Ausfärben mit sauren Farbstoffen (einschließl. der sauer vorzufärbenden Chromierfarben) mehr an Festigkeit und Dauerhaftigkeit verliert als gewöhnliche Wolle, scheint daher immer mehr an Wahrscheinlichkeit zu gewinnen. Die Ergebnisse der eingehenden mikroskopischen Prüfung nicht nur der in der Tabelle (vgl. S. 413) genannten Proben, sondern auch anderer alkalischer und saurer Färbungen könnten mehr oder weniger als Bestätigung dieser Annahme aufgefaßt werden.

Vergleicht man z. B. bei der mikroskopischen Prüfung - wobei man einen Faden aus dem Tuche auszieht, vorsichtig aufdreht und die einzelnen Wollhaare nebeneinander unter Wasser auf den Objektträger ausbreitet - einen mit Indigo in ammoniakalischer Hydrosulfitküpe gefärbten Stoff oder einen mit Indigo vorgeblauten und sauer nachbehandelten Stoff mit einer anderen Stoffprobe, die ohne Indigogrund unmittelbar sauer ausgefärbt wurde, so fällt 